\title{
Variação sazonal e sucessional da comunidade de algas perifíticas em substrato natural em um reservatório mesotrófico tropical
}

\author{
Barbara Golebski Pellegrini ${ }^{1}$ e Carla Ferragut ${ }^{1,2}$
}

Recebido em 13/04/2011. Aceito em 26/06/2012

\begin{abstract}
RESUMO
(Variação sazonal e sucessional da comunidade de algas perifíticas em substrato natural em um reservatório mesotrófico tropical). A colonização e sucessão da comunidade de algas perifíticas em substrato natural (Nymphaea spp.) foi avaliada em um reservatório raso (Lago das Ninféias, PEFI, SP). O estudo avaliou as fontes de variabilidade da estrutura da comunidade perifítica em dois períodos climáticos, contribuindo para o maior entendimento da dinâmica da comunidade em região tropical. A amostragem das variáveis abióticas e bióticas foi realizada a cada três dias até o $12^{\circ}$ dia e cada cinco dias até completar 30 dias no período seco e chuvoso. Os pecíolos de Nymphaea spp. foram aleatoriamente selecionados e limpos. A estrutura do perifíton foi analisada através da clorofila-a, densidade de espécies algais, biovolume, riqueza e diversidade de espécies. O período chuvoso caracterizou-se pelos maiores valores de temperatura e disponibilidade de nutrientes e o período seco pela maior disponibilidade de luz. Os maiores valores de biomassa, densidade e biovolume algal foram observados no período seco. Fases e trajetórias sucessionais distintas foram identificadas em cada período climático. As condições limnológicas mudaram sazonalmente no interior dos bancos de macrófitas e, consequentemente, influenciaram a estrutura da comunidade de algas perifíticas. Concluiu-se que a estrutura da comunidade de algas perifíticas foi sensível às alterações autogênicas (sucessão), mas a escala sazonal foi a principal fonte de variabilidade da estrutura da comunidade.
\end{abstract}

Palavras-chave: biomassa, diversidade, Nymphaea spp., perifíton

\begin{abstract}
(Seasonal and successional variation of a periphytic algal community on natural substrate in a tropical mesotrophic reservoir). Colonization and succession of a periphytic algal community on a natural substrate (Nymphaea spp.) was evaluated in a shallow reservoir (Lago das Ninféias, PEFI, SP). This study aimed to evaluate the environmental factors responsible for the structural variability of a periphytic algal community. Samplings of abiotic and biological variables were carried out every three days up to the 15th day, and later at five days intervals for 30 days in the dry (4/07 to 08/03/2007) and rainy (9/01 to 02/08/2008) seasons. The petioles of Nymphaea spp. were randomly selected and cleaned. Periphytic algal structure was analyzed by measuring chlorophyll-a, algal density, biovolume, species richness and diversity. The rainy season was characterized by high temperature and nutrient availability and the dry season had the highest light availability. Periphyton biomass, algal density and biovolume were higher in the dry season than in rainy season. Different successional phases and trajectories were identified in each climatic period. Limnological conditions inside macrophyte stands changed seasonally, and consequently influenced the periphytic algal structure. The final conclusion is that the periphytic algae structure was sensitive to autogenic changes (succession), but the seasonal scale was the main source of variability in community structure.
\end{abstract}

Key words: biomass, diversity, Nymphaea spp., periphyton

\footnotetext{
${ }^{1}$ Instituto de Botânica, Núcleo de Pesquisa em Ecologia, São Paulo, SP, Brasil
}

${ }^{2}$ Autor para correspondência: carlaferragut@yahoo.com.br 


\section{Introdução}

A comunidade perifítica é um importante produtor primário em lagos, rios e planícies de inundação (Vadeboncoeur et al. 2008, Stevenson 1996), sendo as algas um dos componentes mais abundantes (ex. Kahlert 1998; Frost \& Elser 2002). Considerando os substratos vivos, as algas são importantes colonizadores de macrófitas aquáticas (Wehr \& Sheath 2003). A relação ecológica entre epífitas e macrófitas é bastante controversa, pois interações positivas e negativas são reportadas na literatura (Burkholder 1996; Wetzel 2001). O conhecimento da dinâmica da comunidade perifítica em substrato natural é escasso, principalmente devido ao desconhecimento do tempo de colonização. O maior entendimento da dinâmica da comunidade algal contribuirá para a identificação das possíveis relações ecológicas entre perifíton e macrófita.

A colonização das algas perifíticas inicia-se pelas espécies oportunistas, seguidas por algas com estruturas de aderência e, finalmente, por filamentosas (Hoagland et al. 1982; Lock et al. 1984; Hodoki 2005). O processo de colonização segue para a formação de uma estrutura complexa e estratificada (Hoagland et al. 1982). A dinâmica da comunidade de algas perifíticas pode ser definida pela disponibilidade de local para colonização, pool de propágulos, modos de dispersão e desempenho diferencial das espécies frente à disponibilidade de recursos (Stevenson 1996). A composição estrutural da comunidade depende diretamente dos diferentes desempenhos das espécies que, consequentemente, determinam a trajetória do processo sucessional (Stevenson 1996). Conforme o clássico estudo de Hoagland et al. (1982), a sucessão de algas perifíticas é descrita como uma sequência de eventos que ocorre em um hábitat de espaço limitado, conduzindo a comunidade à "maturidade", complexidade estrutural e tridimensionalidade.

O padrão sucessional de mudanças na fisionomia da comunidade algas perifíticas depende de um complexo conjunto de interações entre as características do hábitat, fatores alogênicos, autogênicos e composição das espécies (Peterson 1996). Estudos mostraram que a trajetória sucessional das espécies no perifíton depende dos fatores ambientais (Rodrigues \& Bicudo 2004; Sekar et al. 2004; Ferragut \& Bicudo 2010). Em ecossistemas lóticos, a trajetória sucessional das algas começa pela colonização pioneira de diatomáceas pequenas e prostradas, seguida por diatomáceas coloniais e clorofíceas filamentosas (Biggs 1996). Esta trajetória sucessional pode não ocorrer em ecossistemas lênticos, pois vários estudos relataram a menor representatividade de diatomáceas como colonizadores pioneiros (ex. Sekar et al. 2004; Rodrigues \& Bicudo 2004; Vercellino \& Bicudo 2006). A sucessão das algas perifíticas em ecossistemas lênticos tropicais é um processo ainda pouco compreendido e a existência de um padrão sucessional é uma questão aberta na ecologia do perifíton.

A maioria dos estudos sobre a colonização e sucessão das algas perifíticas foi desenvolvido em ecossistemas lóticos (ex. McCormick \& Stevenson 1991) e, principalmente, em substrato inerte (ex. Sekar et al. 2004; França et al. 2011). Em relação ao substrato natural, os estudos abordaram as assembléias algais em termos de especificidade (ex. Messyasz \& Kuczynska-Kippen 2006; Cattaneo \& Kalff 1979) e seletividade (Burkholder 1996). Especificamente em ecossistemas lênticos tropicais, os estudos relataram que estruturalmente o perifíton aderido em substrato natural foi predominantemente autotrófico (Leandrini \& Rodrigues 2008), mas a composição de espécies apresentou forte influência da escala sazonal (Cavati \& Fernandes 2008). A influência de fatores ambientais sobre a variação da estrutura da comunidade de epífitas foi relatado em planície de inundação (Rodrigues \& Bicudo 2004; Algarte et al. 2009) e reservatórios (Vercellino \& Bicudo 2006; Cavati \& Fernandes 2008). No Brasil, a trajetória das espécies no processo sucessional da comunidade de algas perifíticas é desconhecida em macrófitas aquáticas.

As mudanças na estrutura da comunidade perifítica podem ser preditivas porque a trajetória sucessional é um processo ordenado, bastante direcional e, ainda, regulado pelas condições ambientais (Odum 2001; McCormick \& Stevenson 1991). Nesse sentido, o conhecimento da trajetória sucessional em termos de substituição de descritores, estratégias adaptativas e atributos da comunidade perifítica podem auxiliar na avaliação da qualidade dos ecossistemas aquáticos. O presente estudo visa analisar o processo de colonização e sucessão da comunidade de algas perifíticas em substrato natural (Nymphaea spp.) em um reservatório mesotrófico tropical (Lago das Ninféias, SP). Primariamente, buscou-se avaliar as fontes de variabilidade da estrutura da comunidade perifítica em dois períodos climáticos, contribuindo para o maior entendimento da dinâmica da comunidade em região tropical.

\section{Material e métodos}

\section{Área de Estudo}

O estudo foi realizado no Lago das Ninféias $\left(23^{\circ} 38^{\prime} \mathrm{S}\right.$, $\left.46^{\circ} 37^{\prime} \mathrm{W}\right)$, o qual está localizado em área preservada de remanescente de Mata Atlântica dentro da área urbana da cidade de São Paulo, o Parque Estadual das Fontes do Ipiranga (PEFI). Lago das Ninféias é formado pelo barramento do córrego Pirarungaua e tem finalidade paisagística no Jardim Botânico de São Paulo. É um ecossistema pequeno, raso e oligo-mesotrófico com área de $5.433 \mathrm{~m}^{2}$, profundidade máxima de $3,6 \mathrm{~m}$, média de $1,32 \mathrm{~m}$ e tempo de residência de 7,2 dias (Bicudo et. al. 2002). O Lago das Ninféias apresenta uma região litorânea com elevada heterogeneidade de hábitat decorrente da grande diversidade de macrófitas aquáticas (Bicudo et al. 2002), sendo Nymphaea spp. e Utricularia foliosa as mais abundantes.

\section{Delineamento amostral}

A colonização do perifíton desenvolvido no pecíolo da Nymphaea spp. foi acompanhada durante 30 dias no período 
seco (04/07 a 03/08/2007) e chuvoso (9/01 a 8/02/2008) nos intervalos de 3, 6, 9, 12, 15, 20, 25 e 30 dias. No período chuvoso não foi possível realizar amostragem no $30^{\circ}$ dia devido ao adiantado processo de senescência dos pecíolos selecionados para amostragem. No início do período amostral $\left(\mathrm{T}_{0}\right)$, as macrófitas com folhas de tamanho semelhante foram selecionadas e, identificadas com fio de náilon (inerte). Os pecíolos das macrófitas selecionadas foram limpos utilizando gazes esterilizadas. Foram limpos cerca de 30 pecíolos, os quais foram coletados aleatoriamente através de sorteio. Conjuntamente com a amostragem do perifíton foram coletadas amostras de água circundante ao substrato para amostragem das variáveis físicas e químicas.

\section{Variáveis analisadas}

As variáveis climatológicas avaliadas foram: temperatura do ar e precipitação pluviométrica. Os dados meteorológicos foram fornecidos pela Estação Meteorológica do Instituto Astronômico e Geofísico da Universidade de São Paulo. Foram analisadas as seguintes variáveis abióticas: radiação subaquática (Licor, modelo LI-205), temperatura da água, alcalinidade (Golterman \& Clymo 1971), formas de carbono inorgânico (Mackeret et al. 1978), pH (pHmetro 20 Digimed), condutividade elétrica (condutivímetro Digimed), oxigênio dissolvido (Golterman et al. 1978), nitrogênio total e fósforo total (Valderrama 1981).

O material perifítico aderido ao pecíolo das ninféias foi retirado através de raspagem com escova dental de cerdas macias e, subsequentemente, lavado com água destilada. A biomassa foi analisada através da clorofila-a (corrigida da feofitina) conforme Marker et al. (1980) e Sartory \& Grobblelar (1984). A análise taxonômica da comunidade de algas perifíticas foi realizada em microscópio binocular Zeiss Axioplan 2 a partir de amostras fixadas com solução de formalina $4 \%$. Lâminas permanentes foram feitas para a identificação das diatomáceas. Para análise quantitativa, as amostras foram fixadas em lugol acético e a contagem realizada em microscópio invertido Zeiss Axiovert (400x) conforme Utermöhl (1958). O limite de contagem foi estabelecido de acordo com a curva de rarefação de espécies e até atingir 100 indivíduos da espécie mais abundantes (Bicudo 1990). O biovolume algal foi obtido pela multiplicação da densidade de cada espécie pelo volume médio de suas células (Hillebrand et al. 1999). Para análise das descritoras da comunidade foram consideradas as espécies com representatividade maior do que 5\% da densidade total em cada de dia de colonização. $O$ índice de dominância de Simpson foi utilizado como medida da estrutura da comunidade (Odum 2001). Foram determinadas as espécies dominantes e abundantes (Lobo \& Leighton 1986).

\section{Tratamento Estatístico}

Para análise inferencial aplicou-se a análise de variância (ANOVA-1 fator) para a comparação das variáveis ambientais entre períodos climáticos $(\alpha=0,05)$. A similaridade taxonômica da comunidade de algas perifíticas durante a sucessão foi realizada através da análise de agrupamento, usando associação média (UPGMA) e índice de Bray-Curtis. A análise conjunta das variáveis abióticas e bióticas foi realizada através da análise de correspondência canônica (CCA) com matriz de covariância e dados transformados pela amplitude de variação $\left(\left[\left(\mathrm{X}-\mathrm{X}_{\min }\right) /\left(\mathrm{X}_{\max }-\mathrm{X}_{\min }\right)\right]\right.$. Foram considerados os táxons com abundância relativa maior ou igual a $1 \%$. Os programas estatísticos utilizados foram PC-ORD (McCune \& Mefford 1999) e MINITAB 14.0.

\section{Resultados}

\section{Variáveis Climatológicas}

No período seco a temperatura do ar apresentou média mensal de $15,6^{\circ} \mathrm{C}$ e no chuvoso de $20,9^{\circ} \mathrm{C}$. A precipitação pluviométrica mensal média foi de $106,9 \mathrm{~mm}$ no período seco e de $237,0 \mathrm{~mm}$ no chuvoso.

\section{Variáveis Limnológicas}

A tabela 1 resume as condições físicas e químicas da água dentro do banco de ninféias no período de estudo. Os valores de radiação subaquática e a temperatura da água foram maiores no período chuvoso, mas a disponibilidade de luz foi maior no período seco. A condutividade elétrica, alcalinidade e o $\mathrm{pH}$ apresentaram valores médios muito similares no período seco e chuvoso. Em relação aos gases dissolvidos, verificou-se que a disponibilidade de $\mathrm{CO}_{2}$ livre foi maior no período seco, mas variação também foi elevada. Contrapondo, os menores teores de OD foram registrados no período seco. Considerando os nutrientes totais, verificou-se que as maiores concentrações de NT e PT foram registradas no período chuvoso. Contudo, o coeficiente de variação foi elevado para o PT e baixo para o NT.

A análise de variância (Anova 1-fator) mostrou que apenas os valores de radiação subaquática $(\mathrm{F}=7,536, p=0,013)$, a temperatura da água ( $\mathrm{F}=44,566, p=0,0004)$ e a concentração de PT $(\mathrm{F}=68,421, p=0,0009)$ foram significativamente diferentes entre os períodos climáticos.

\section{Comunidade Perifítica}

Considerando os atributos da comunidade perifítica, verificou-se que o incremento de biomassa e o crescimento algal (densidade e biovolume) aumentaram exponencialmente no período seco e chuvoso (Fig. 1). No período seco o valor máximo de densidade, biomassa e biovolume ocorreram no $15^{\circ}, 20^{\circ}$ e $25^{\circ}$ estádio sucessional, respectivamente. No período chuvoso o valor máximo de densidade e biovolume foram atingidos no $20^{\circ}$ estádio sucessional, enquanto o da biomassa foi no $15^{\circ}$ estádio. Em ambos os períodos climáticos houve redução do crescimento algal (Seca: 77,8\%; Chuva: 51\%) e perda de biomassa (Seca: 43,5\%; Chuva: 90,6\%) nos últimos dias de colonização.

$\mathrm{Na}$ análise qualitativa da comunidade perifítica foi identificado um total de 259 táxons, tendo no período seco um 
Tabela 1. Valor mínimo e máximo e, entre parênteses valor médio, desvio padrão e coeficiente de variação (\%) das variáveis abióticas ( $\mathrm{n}=9$ ) analisadas no Lago das Ninféias no período seco e chuvoso.

\begin{tabular}{lcc}
\hline Variáveis & Período Seco & Período Chuvoso \\
\hline Alcalinidade $\left(\mathrm{mEq} \cdot \mathrm{L}^{-1}\right)$ & $0,09-0,23(0,1 \pm 0,0025 ; 25,4 \%)$ & $0,21-0,27(0,25-0,02 ; 7,7 \%)$ \\
Condutividade elétrica $\left(\mu \mathrm{S} . \mathrm{cm}^{-1}\right)$ & $49,2-57,2(51,7 \pm 5,2 ; 2,5 \%)$ & $50,6-55,9(53,6 \pm 4,0 ; 2,1 \%)$ \\
Gás Carbônico Livre $\left(\mu \mathrm{S} . \mathrm{cm}^{-1}\right)$ & $6,1-119,7(23,2-168,5 ; 36,6 \%)$ & $6,5 \pm 16,0(9,7-29,5 ; 2,9 \%)$ \\
Fósforo Total $\left(\mu \mathrm{g} . \mathrm{L}^{-1}\right)$ & $85,91-580,91(282,2 \pm 217,8 ; 77,3 \%)$ & $249,5-482,8(345,4 \pm 84,3 ; 24,4 \%)$ \\
Oxigênio dissolvido $\left(\mathrm{mg} . \mathrm{L}^{-1}\right)$ & $0,6-5,8(4,0 \pm 1,5 ; 39,5 \%)$ & $3,7-5,3(4,7 \pm 0,5 ; 11,3 \%)$ \\
pH & $4,9-6,6(6,2 \pm 8,9 ; 0,5 \%)$ & $6,2-6,6(6,4 \pm 1,8 ; 0,1 \%)$ \\
Nitrogênio Total $\left(\mu \mathrm{g} . \mathrm{L}^{-1}\right)$ & $11,4-17,0(14,1 \pm 2,1 ; 15,2 \%)$ & $19,3-32,8(24,8 \pm 4,7 ; 19,0 \%)$ \\
Radiação subaquática $\left(\mu \mathrm{mol} \cdot \mathrm{m}^{-2} . \mathrm{s}^{-1}\right)$ & $47,2-512,8(231,6 \pm 177,3 ; 76,6 \%)$ & $26,6-884,9(415,8 \pm 69,1-287,2 \%)$ \\
Razão molar NT:PT & $8,9-56,4(25,2 \pm 17,7 ; 68 \%)$ & $41,3-76,2(54,4 \pm 7,5 ; 14 \%)$ \\
Temperatura da água $\left({ }^{\circ} \mathrm{C}\right)$ & $12,0-18,0(15,7 \pm 168,5 ; 1,8 \%)$ & $20,0-26,0(23,0 \pm 29,5 ; 2,1 \%)$ \\
\hline
\end{tabular}
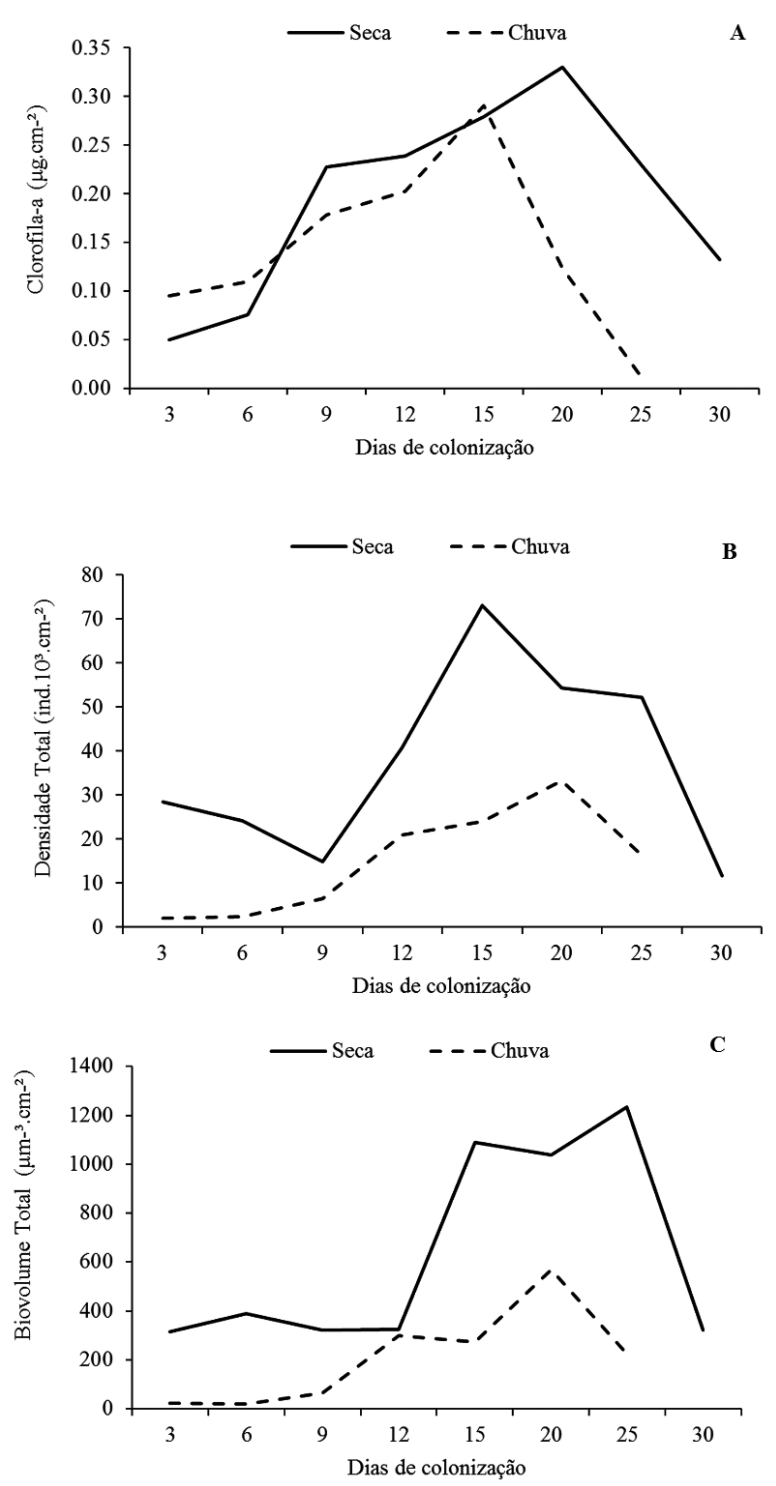

Figura 1. Variação temporal da clorofila-a $(A ; n=2)$, densidade (B) e biovolume total (C) da comunidade de algas perifíticas no período seco e chuvoso. total de 160 táxons e de 133 no chuvoso. No período seco, as espécies foram distribuídas em onze classes taxonômicas, sendo Chlorophyceae a classe de maior número de espécies. No período chuvoso, as espécies foram distribuídas em treze classes taxonômicas, sendo Zygnemaphyceae a classe de maior número de espécies.

A densidade relativa de classes algais variou em escala sazonal, contudo Bacillariophyceae e Chlorophyceae apresentaram grande participação na estrutura da comunidade em ambos os períodos climáticos (Fig. 2). Considerando a variação sucessional no período seco, verificou-se que Cyanophyceae (22-34\%) e Chlorophyceae (24-30\%) apresentaram elevada representatividade nos estádios iniciais da sucessão, mas com o avanço houve aumento da participação de Bacillariophyceae e a drástica redução de Cyanophyceae (Fig. 2). No período chuvoso, Chlorophyceae (24-38\%) e Chrysophyceae (18-29\%) apresentaram elevada participação na estrutura da comunidade nos estádios iniciais e com avanço da sucessão ocorreu aumento de Zygnemaphyceae e Bacillariophyceae. A maior participação de Zygnemaphyceae ocorreu no $20^{\circ}$ estádio. Bacillariophyceae apresentou crescimento exponencial ao longo da sucessão em ambos os períodos climáticos, mas a maior densidade ocorreu no $25^{\circ}$ estádio.

A similaridade taxonômica da comunidade de algas perifíticas entre os estádios sucessionais foi evidenciada pela análise de agrupamento (Fig. 3). No período seco o grau de similaridade da comunidade algal nos estádios iniciais ( $3^{\circ}, 6^{\circ}$ e o $9^{\circ}$ estádio) foi de $60 \%$ e nos estádios avançados de $54 \%$ ( $12^{\circ}$ ao $25^{\circ}$ estádio). A comunidade no $30^{\circ}$ estádio apresentou a menor similaridade com os demais estádios sucessionais (32\%). No período chuvoso a comunidade do $3^{\circ}$ e $6^{\circ}$ estádio apresentou similaridade de $52 \%$ e dos estádios avançados de $59 \%$ ( $12^{\circ}-20^{\circ}$ estádio). A similaridade taxonômica foi maior entre o $9^{\circ}$ e o $25^{\circ}$ estádio, os quais apresentaram a menor similaridade com os demais estádios sucessionais (54\%). A análise de grupamento mostrou as fases sucessionais da comunidade de algas perifíticas. 

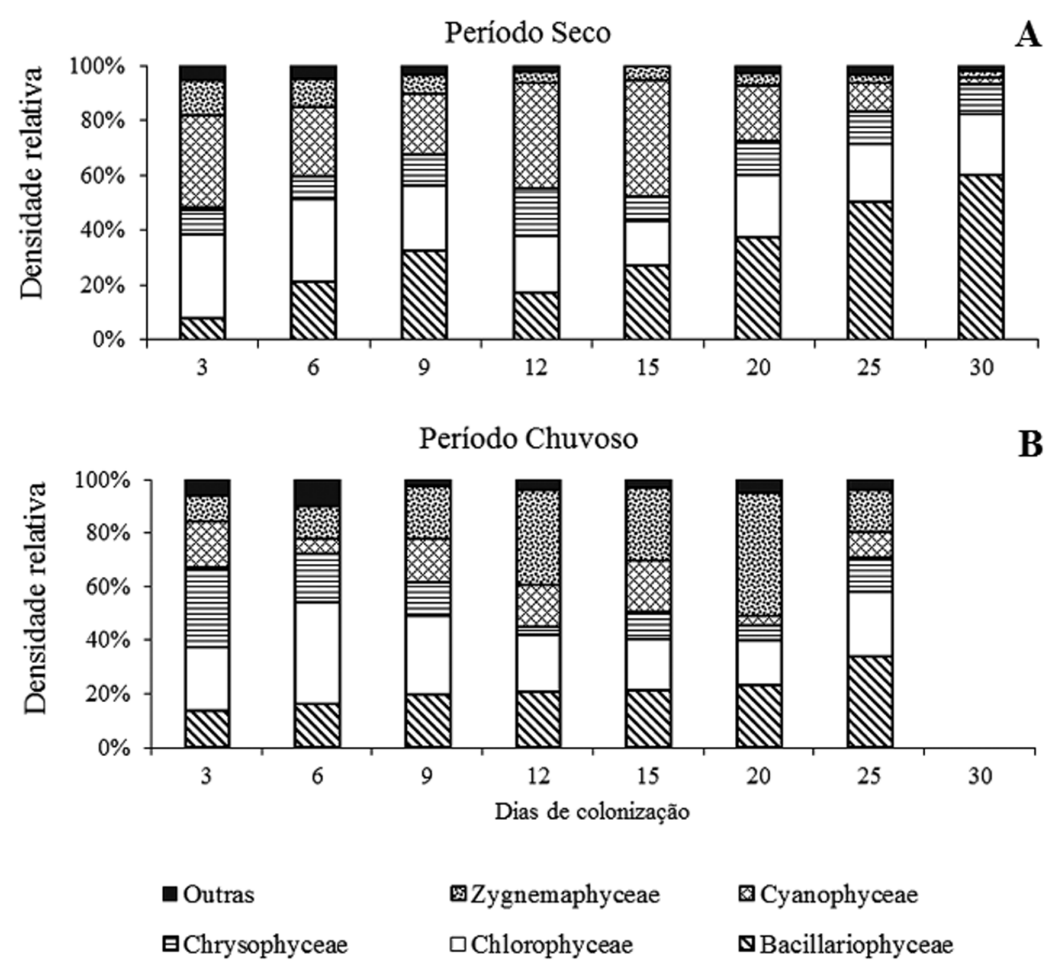

Figura 2. Densidade relativa de classe de algais no perifíton em substrato natural no Lago das Ninféias no período seco (A) e chuvoso (B).

Período Seco (A)

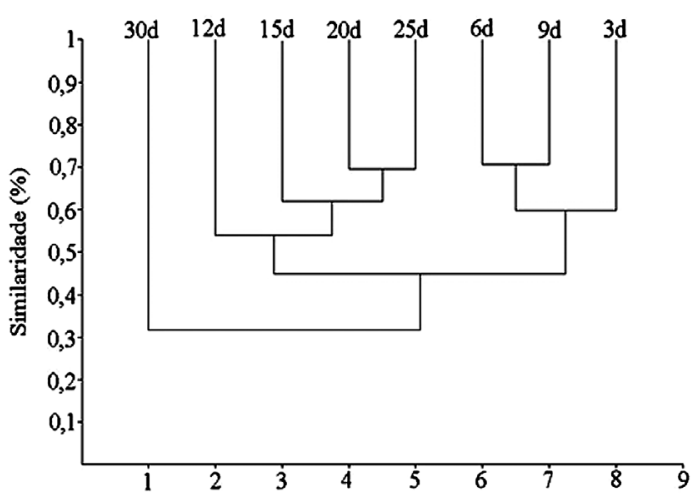

Período Chuvoso (B)

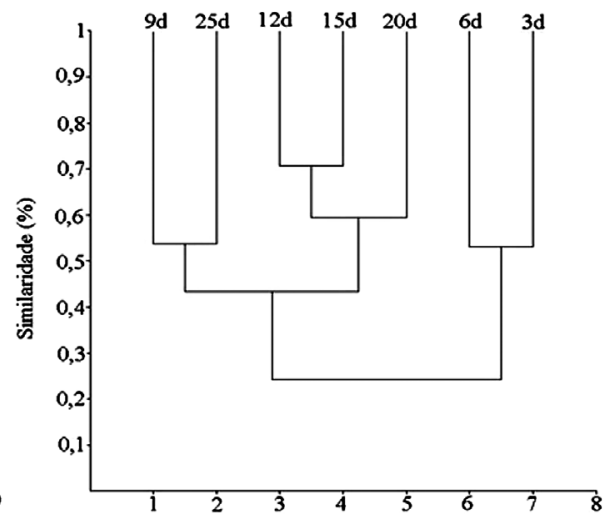

Figura 3. Análise de agrupamento calculada por associação média (UPGMA) e pelo índice de Bray-Curtis das algas do perifíton no período seco (A) e no chuvoso (B).

Em ambos os períodos climáticos, nenhuma espécie foi classificada como dominante durante o processo sucessional. Entretanto, observou-se a ocorrência de 46 espécies abundantes no período seco e 33 no chuvoso. Synechocystis aquatilis e Frustulia crassinervia foram as principais descritoras da comunidade no período seco, representando em média de $22,2 \%$ e $14,4 \%$ da densidade total no período de estudo. Cosmarium blyttii apresentou a maior contribuição para a densidade total (média 20,8\%) no período chuvoso (Fig. 4).

Considerando a substituição das descritoras na comunidade perifítica durante a colonização, verificou-se que a sequência das espécies na sucessão foi diferente nos períodos climáticos (Fig. 4, Tab. 2). No período seco os estádios iniciais apresentaram alta participação de Synechocystis aquatilis (24\%) e Chlamydomonas sordida (13\%). Apesar da elevada participação de S. aquatilis na estrutura, observou-se o aumento exponencial de Frustulia crassinervia e Navicula cryptotenella durante a sucessão. No $30^{\circ}$ estádio ocorreu forte declínio populacional de $S$. aquatilis e aumento da participação das diatomáceas.

No período chuvoso ocorreu elevada participação de flagelados nos estádios iniciais da sucessão, principalmente de 
Chlamydomonas sagittula, Chromulina elegans e Chlamydomonas gloeopara (Fig. 4, Tab. 2). No intervalo sucessional de 9 a 20 dias houve aumento da participação de Zygnemaphyceae, principalmente Cosmarium blyttii (14-30,8\%). No $25^{\circ}$ estádio houve redução do crescimento algal, mas a densidade populacional de Eunotia bilunaris aumentou.

O maior valor da diversidade de espécies foi registrado no $15^{\circ}$ estádio do período seco e no $20^{\circ}$ do período chuvoso. Em média, a diversidade foi maior no período seco do que no chuvoso (Tab. 2).

A CCA apresentou autovalores para o eixo $1(\lambda=0,365)$ e $2(\lambda=0,122)$ que explicaram $14,1 \%$ da variabilidade dos dados (Fig. 5, Tab. 3-4). A elevada correlação espécie-ambiente de Pearson para o eixo $1(r=0,942)$ e $2(r=0,958)$ indicou forte relação entre a distribuição das espécies e as variáveis ambientais. O teste Monte Carlo de permutação demonstrou que a correlação entre as espécies de algas perifíticas e as condições ambientais foram estatisticamente significativas nos eixos 1 e $2(p=0,02)$.

As variáveis ambientais correlacionadas com eixo 1 descreveram um gradiente sazonal. Os coeficientes canônicos mostraram que temperatura foi a variável ambiental mais importante na ordenação do eixo 1 e de NT para o eixo 2 (Tab. 3). No lado negativo do eixo foram ordenadas as unidades amostrais do período de seca, as quais foram associadas aos maiores valores de radiação subaquática. Em oposição, as unidades do período chuvoso foram correlacionadas positivamente com os maiores valores de temperatura e fósforo total.

Claramente, o eixo 1 mostrou que a escala sazonal pesou na ordenação das espécies no eixo, evidenciando uma sequência sucessional específica para cada período climático. De acordo a propriedade centróide da CCA, verificou-se que o maior número de espécies foram as mais associadas ao $15^{\circ}$ e $20^{\circ}$ estádios da sucessão no período seco, tendo cinco espécies alta correlação com o eixo $(r>0,6$; Chromulina pygmaea, Frustulia crassinervia, Navicula cryptotenella, Pseudanabaena galeata, Pseudodidymocystis fina). No período chuvoso, as espécies Mougeotia sp., Eunotia flexuosa var.flexuosa e Chlamydomonas gloeopara foram as espécies mais associadas aos estádios avançados $\left(15^{\circ}-20^{\circ}\right)$.

\section{Discussão}

O Lago das Ninféias é um ecossistema raso e com extensos bancos de macrófitas aquáticas na região litorânea
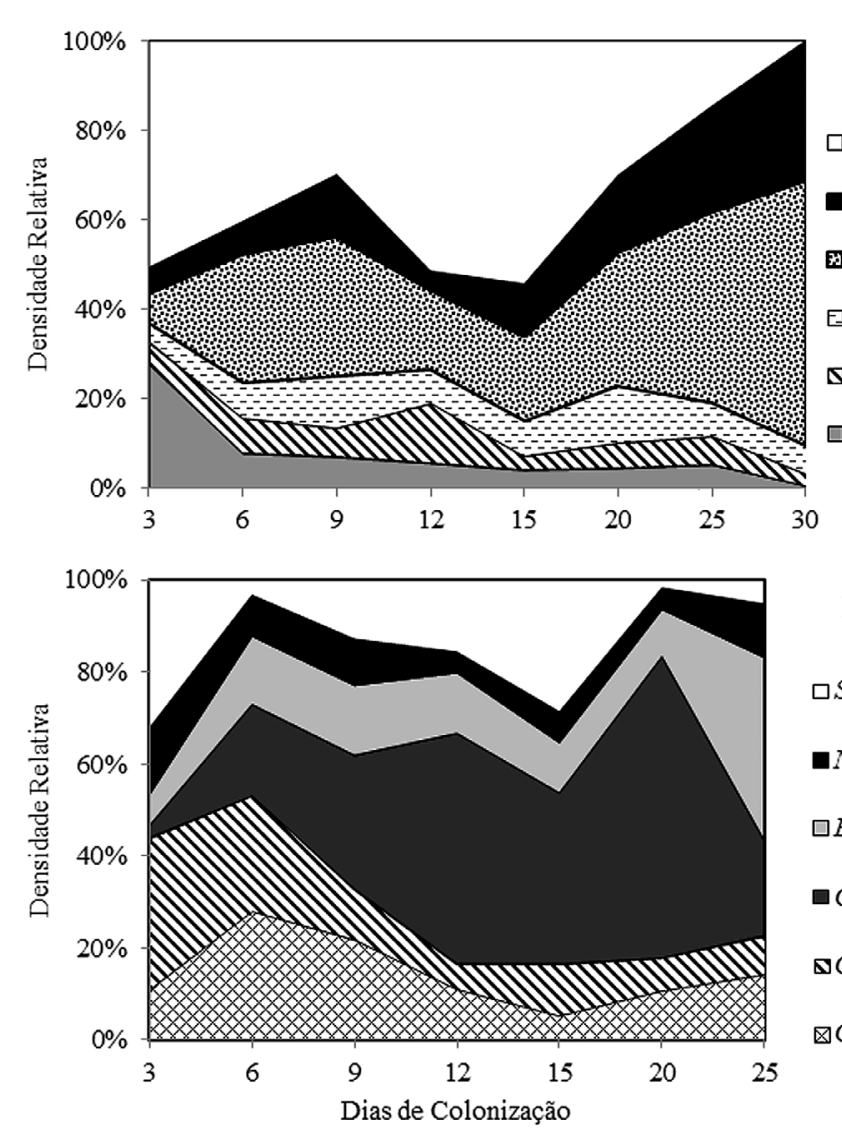

A

Período Seco

$\square$ Synechocystis aquatilis

Navicula cryptotenella

团Frustulia crassinervia

घChromulina pygmaea

$\Delta$ Chromulina elegans

$\square$ Chlamydomonas sordida

B

\section{Período Chuvoso}

$\square$ Synechocystis aquatilis

- Navicula cryptotenella

$\square$ Eunotia bilunaris

- Cosmarium blyttii

sChromulina elegans

凶Chlamydomonas gloeopara

Figura 4. Variação sucessional das descritoras da comunidade de algas perifíticas em substrato natural no Lago das Ninféias no período seco(A) e chuvoso (B). 
Tabela 2. Síntese dos principais atributos e espécies descritoras do perifíton utilizados na caracterização das fases sucessionais das algas perifíticas em substrato natural (Nymphaea spp.).

\begin{tabular}{|c|c|c|c|c|c|c|c|c|c|}
\hline \multicolumn{10}{|c|}{ PERÍODO SECO } \\
\hline & \multirow{2}{*}{ Tempo de colonização } & \multicolumn{3}{|c|}{ Fase I } & \multicolumn{4}{|c|}{ Fase II } & \multirow{2}{*}{$\begin{array}{c}\text { Fase III } \\
30\end{array}$} \\
\hline & & 3 & 6 & 9 & 12 & 15 & 20 & 25 & \\
\hline Descritoras & Chlamydomonas sordida & 13 & 3 & 4 & 3 & 3 & 2 & 2 & 0 \\
\hline \multirow[t]{3}{*}{ (\%) } & Frustulia crassinervia & 3 & 11 & 16 & 11 & 13 & 16 & 21 & 26 \\
\hline & Navicula cryptotenella & 3 & 3 & 7 & 3 & 8 & 9 & 12 & 14 \\
\hline & Synechocystis aquatilis & 24 & 16 & 16 & 31 & 37 & 16 & 7 & 0 \\
\hline Classes & Bacillariophyceae & 8 & 21 & 32 & 17 & 27 & 37 & 50 & 60 \\
\hline \multirow[t]{3}{*}{$(\%)$} & Chlorophyceae & 30 & 30 & 24 & 21 & 16 & 23 & 21 & 22 \\
\hline & Cyanophyceae & 34 & 25 & 22 & 39 & 42 & 20 & 10 & 2 \\
\hline & Zygnemaphyceae & 13 & 10 & 7 & 4 & 5 & 5 & 3 & 3 \\
\hline \multirow[t]{3}{*}{ Atributos } & Densidade Total (ind $10^{3} \cdot \mathrm{cm}^{-2}$ ) & 28 & 24 & 15 & 41 & 73 & 54 & 52 & 12 \\
\hline & Biomassa $\left(\mu \mathrm{g} . \mathrm{cm}^{-2}\right)$ & 0,1 & 0,1 & 0,2 & 0,2 & 0,3 & 0,3 & 0,2 & 0,1 \\
\hline & Biovolume Total $\left(10^{6} \mu \mathrm{m}^{3} \cdot \mathrm{cm}^{-2}\right)$ & 32 & 39 & 32 & 32 & 109 & 104 & 124 & 32 \\
\hline Diversidade & Índice de Dominância & 0,09 & 0,06 & 0,07 & 0,12 & 0,16 & 0,07 & 0,08 & 0,10 \\
\hline \multicolumn{10}{|c|}{ PERÍODO CHUVOSO } \\
\hline & \multirow{2}{*}{ Tempo de colonização } & \multicolumn{3}{|c|}{ Fase I } & \multicolumn{4}{|c|}{ Fase II } & Fase III \\
\hline & & 3 & 6 & & 9 & 12 & 15 & 20 & 25 \\
\hline Descritoras & Chlamydomonas gloeopara & 5 & 12 & & 10 & 5 & 2 & 5 & 6 \\
\hline \multirow[t]{5}{*}{ (\%) } & Chlamydomonas sagittula & 11 & 2 & & 1 & 1 & 3 & 1 & 1 \\
\hline & Chromulina elegans & 14 & 11 & & 5 & 2 & 5 & 3 & 4 \\
\hline & Cosmarium blyttii & 1 & 9 & & 14 & 21 & 16 & 31 & 9 \\
\hline & Eunotia bilunaris & 3 & 7 & & 7 & 6 & 5 & 5 & 18 \\
\hline & Synechocystis aquatilis & 13 & 1 & & 6 & 7 & 13 & 1 & 2 \\
\hline Classes & Bacillariophyceae & 14 & 16 & & 20 & 21 & 19 & 23 & 33 \\
\hline \multirow[t]{4}{*}{ (\%) } & Chlorophyceae & 24 & 38 & & 29 & 21 & 18 & 16 & 24 \\
\hline & Chrysophyceae & 29 & 18 & & 12 & 3 & 9 & 6 & 12 \\
\hline & Cyanophyceae & 17 & 6 & & 16 & 15 & 17 & 4 & 9 \\
\hline & Zygnemaphyceae & 10 & 12 & & 19 & 35 & 25 & 46 & 16 \\
\hline \multirow[t]{3}{*}{ Atributos } & Densidade Total (ind $10^{3} \cdot \mathrm{cm}^{-2}$ ) & 2 & 2 & & 6 & 21 & 24 & 33 & 16 \\
\hline & Biomassa $\left(\mu \mathrm{g} . \mathrm{cm}^{-2}\right)$ & 0,1 & 0,1 & & 0,2 & 0,2 & 0,3 & 0,1 & 0,0 \\
\hline & Biovolume Total $\left(10^{6} \mu \mathrm{m}^{3} \cdot \mathrm{cm}^{-2}\right)$ & 2 & 2 & & 6 & 30 & 27 & 57 & 23 \\
\hline Diversidade & Îndice de Dominância & 0,07 & 0,06 & & 0,06 & 0,07 & 0,06 & 0,11 & 0,06 \\
\hline
\end{tabular}

Tabela 3. Coeficientes canônicos e correlações de Pearson das variáveis ambientais com os eixos 1 e 2.

\begin{tabular}{|c|c|c|c|c|}
\hline \multirow[b]{2}{*}{ Variáveis } & \multicolumn{2}{|c|}{ Coeficiente canônico } & \multicolumn{2}{|c|}{ Correlação de Pearson } \\
\hline & Eixo 1 & Eixo 2 & Eixo 1 & Eixo 2 \\
\hline Gás carbônico livre & -0.371 & -0.586 & 0,847 & $-0,01$ \\
\hline Fósforo Total (PT) & 0.154 & -0.501 & 0,256 & 0,923 \\
\hline Nitrogênio Total (NT) & 0.329 & 1.001 & 0,912 & $-0,207$ \\
\hline Penetração de luz & -0.388 & -0.062 & $-0,812$ & 0,042 \\
\hline Temperatura & 0.561 & -0.248 & $-0,624$ & 0,180 \\
\hline
\end{tabular}




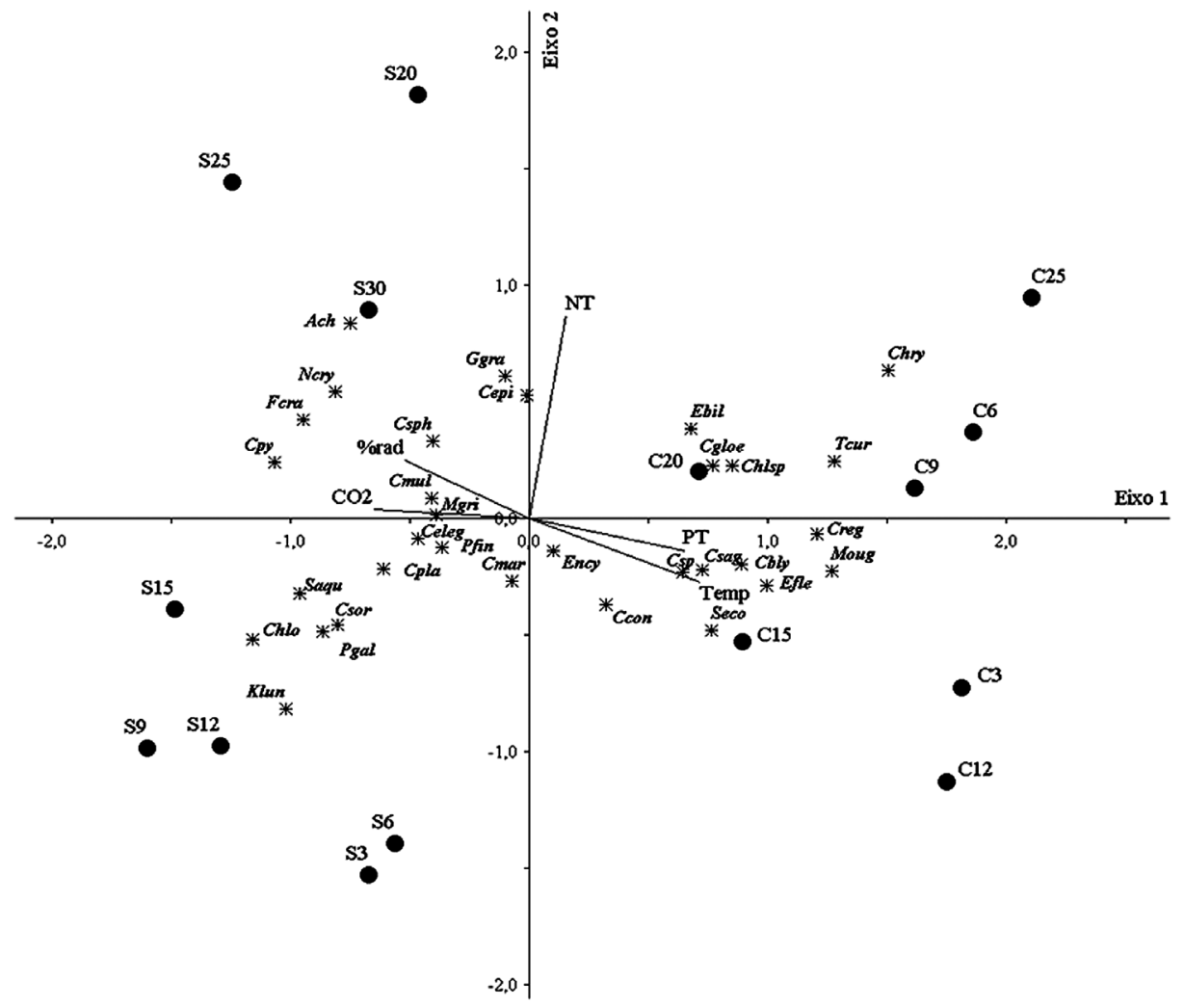

Figura 5. CCA da densidade das algas perifíticas descritoras em substrato natural no Lago das Ninféias no período seco e chuvoso. Abreviações: Unidades Amostrais: $\mathrm{S}=$ seca; $\mathrm{C}=$ chuva, número representa o tempo de colonização; Vetores: $\mathrm{NT}=$ nitrogênio total; $\mathrm{PT}=$ fósforo total; $\% \mathrm{Rad}=$ porcentagem de radiação subaquática; $\mathrm{Temp}=$ temperatura; $\mathrm{CO} 2=$ $\mathrm{CO}_{2}$ livre. Códigos das espécies encontram-se na tabela 4.

(Bicudo et al. 2002). É um ecossistema bastante favorável ao desenvolvimento da comunidade perifítica, pois as macrófitas aquáticas promovem uma ampla diversidade de hábitat. As macrófitas podem modificar o hábitat circundante, atuar sobre as propriedades dos substratos e inter-relações ecológicas (Jeppesen et al. 1998). No presente estudo as condições limnológicas no interior dos bancos de ninféias foram distintas entre os períodos climáticos. A condição limnológica no período chuvoso caracterizou-se, principalmente, pela maior temperatura e disponibilidade de nutrientes e no período seco pela maior disponibilidade de luz. Nestas condições ambientais, encontrou-se no substrato natural (Nymphaea spp.) um processo de colonização e sucessão da comunidade de algas perifíticas específico para cada período climático.

O tempo de colonização de duas a quatro semanas é considerado o intervalo ideal para a biomassa atingir o valor máximo em rios e lagos de região temperada e tropical (ex. Cattaneo \& Amireault 1992; Ács \& Kiss 1993; Bicudo et al. 1995). A comunidade de algas perifíticas apresentou aumento exponencial da biomassa e densidade total entre o $15^{\circ}$ e $20^{\circ}$ estádio sucessional em ambos os períodos climáticos. Em região tropical, estudos reportaram o pico de biomassa e crescimento algal em substrato artificial no intervalo de 20 a 30 dias de colonização (ex. Vercellino \& Bicudo 2006; França et al. 2011). Apesar das modificações na arquitetura da macrófita durante seu crescimento e senescência (Carpenter \& Lodge 1986), presentemente, o pico de biomassa e crescimento algal foi atingindo dentro do tempo de colonização descrito na maioria dos estudos.

Em termos de incremento de biomassa e crescimento algal, o período seco foi mais favorável ao desenvolvimento do perifíton. Comparativamente, a disponibilidade de luz no interior dos bancos de macrófitas foi maior no período seco, o que pode ter favorecido o maior desenvolvimento do perifíton. Fato explicado pela visível redução da porcentagem de cobertura dos bancos de macrófitas no Lago das Ninféias, os quais podem ser reduzidos a menos de $20 \%$ no inverno (dados não publicados). Além de aumentar a disponibilidade de luz, a diminuição da cobertura de macrófitas pode ter reduzido a competição por nutrientes, o que muito provavelmente, também deve ter contribuído para o maior desenvolvimento do perifíton. Cattaneo et al. (1998) notaram que as diferenças na intensidade de luz nos diferentes tipos de vegetação podem afetar o incremento de biomassa perifítica. Estudos anteriores na área do estudo (PEFI) reportaram o maior desenvolvimento do perifíton no período seco (Vercellino \& Bicudo 2006; Borduqui et 
Tabela 4. Correlação de Pearson da densidade das de algas perifíticas $(n=32)$ com os eixos 1 e 2 da CCA.

\begin{tabular}{|c|c|c|c|}
\hline & Códigos & Eixo 1 & Eixo 2 \\
\hline Achnantes sp. & Ach & $-0,440$ & 0,598 \\
\hline Carteria multifilis (Fresenius) Dill & Cmul & $-0,499$ & 0,234 \\
\hline Chlamydomonas epiphytica G.M. Smith & Cepi & $-0,123$ & 0,549 \\
\hline Chlamydomonas gloeopara Rodhe \& Skuja & Cgloe & 0,469 & 0,373 \\
\hline Chlamydomonas planctogloea Skuja & Cpla & $-0,497$ & $-0,078$ \\
\hline Chlamydomonas sagittula Skuja & Csag & 0,282 & $-0,065$ \\
\hline Chlamydomonas sordida Ettl & Csor & $-0,574$ & $-0,255$ \\
\hline Chlamydomonas sp. & Chlsp & 0,347 & 0,241 \\
\hline Chlorella cf. & Chlo & $-0,317$ & $-0,124$ \\
\hline Chromulina elegans Doflein & Celeg & $-0,512$ & 0,040 \\
\hline Chromulina pygmaea Nygaard & Cpy & $-0,669$ & 0,259 \\
\hline Chromulina sphaerica Bachmann & Csph & $-0,399$ & 0,410 \\
\hline Chrysophyceae sp. & Chry & 0,469 & 0,356 \\
\hline Cosmarium blyttii Wille & Cbly & 0,307 & $-0,041$ \\
\hline Cosmarium contractum Kirchner. var. contractum & Ccon & 0,088 & $-0,211$ \\
\hline Cosmarium margaritatum (Lundell) Roy \& Bisset var margaritatum f. minor (Boldt) West \& West & Cmar & $-0,135$ & $-0,103$ \\
\hline Cosmarium regnellii Wille & Creg & 0,425 & 0,032 \\
\hline Cosmarium sp. & Csp & 0,314 & $-0,097$ \\
\hline Encyonema sp. & Ency & $-0,059$ & $-0,015$ \\
\hline Eunotia bilunaris (Ehrenberg) Schaarschmidt & Ebil & 0,370 & 0,505 \\
\hline Eunotia flexuosa (Brébisson) Kützing var. flexuosa & Efle & 0,488 & $-0,133$ \\
\hline Frustulia crassinervia (Brébisson) Lange-Bertalot \& Krammer & Fcra & $-0,700$ & 0,447 \\
\hline Gomphonema gracile Ehrenberg & Ggra & $-0,195$ & 0,666 \\
\hline Kirchneriella lunaris (Kirchner) Möbius & Klun & $-0,467$ & $-0,357$ \\
\hline Monoraphidium griffithii (Berkeley) Komárková-Legnerová & Mgri & $-0,362$ & 0,114 \\
\hline Mougeotia sp. & Moug & 0,512 & $-0,063$ \\
\hline Navicula cryptotenella Lange-Bertalot & Ncry & $-0,584$ & 0,518 \\
\hline Pseudanabaena galeata Böcher & Pgal & $-0,728$ & $-0,330$ \\
\hline Pseudodidymocystis fina (Komárek) E.Hegewald \& Deason & Pfin & $-0,573$ & 0,000 \\
\hline Scenedesmus ecornis (Ehrenberg) Chodat & Seco & 0,311 & $-0,253$ \\
\hline Synechocystis aquatilis Sauvageau & Saqu & $-0,583$ & $-0,133$ \\
\hline Trachelomonas curta Cunha & Tcur & 0,462 & 0,207 \\
\hline
\end{tabular}

al. 2008; Oliveira et al. 2010). Diferentes fatores ambientais foram associados ao maior desenvolvimento do perifíton no período seco, como a diminuição da floração de cianobactérias (Borduqui et al. 2008; Oliveira et al. 2010) e a maior disponibilidade de nutrientes (Vercellino \& Bicudo 2006).

A densidade total e a representatividade das classes algais na estrutura da comunidade perifítica no pecíolo das ninféias apresentou influência direta da escala sazonal. Apesar da alta representatividade de Bacillariophyceae na comunidade perifítica em ambos os períodos, a dominância da classe ocorreu somente no período seco. Estudos anteriores reportaram a dominância de diatomáceas perifíticas no período seco em reservatório tropical hipereutrófico
(Borduqui et al. 2008; Ferrari 2010). Uma importante descritora foi a $r$-estrategista Frustulia crassinervia (sensu Biggs et al. 1998), a qual é associada à condição oligotrófica (Van Dam et al. 1984). Ferrari (2010) comenta que a F. crassinervia tenha possivelmente uma amplitude ecológica maior do que a registrada até o momento, pois a espécie possui ampla distribuição geográfica e ocorrência no perifíton e fitoplâncton. Outra importante classe no período seco foi Cyanophyceae, a qual teve alta representatividade nos estádios iniciais. A participação de cianobactérias no início do processo sucessional é comumente relatada em região tropical (ex. França et al. 2011, Cavati \& Fernandes 2008). Synechocystis aquatilis foi uma eficiente colonizadora, sendo 
descritora nos estádios iniciais e intermediários da sucessão. Esta espécie é comumente encontrada dentre as descritoras no perifíton de região tropical (França et al. 2011; Fonseca \& Rodrigues 2007). A ocorrência de S. aquatilis pode ser explicada pela eficiência de suas estratégias adaptativas de sobrevivência, tais como a elevada razão superfície-volume, eficiente assimilação de nutrientes e habilidade competitiva em baixa intensidade luminosa (Callieri \& Stockner 2002). Na área de estudo, S. aquatilis foi encontrada como dominante nos estádios iniciais da sucessão em reservatório hipereutrófico (Borduqui \& Ferragut 2012) e, ainda, durante toda sucessão em experimento de pulsos de enriquecimentos em reservatório mesotrófico (Vercellino 2007).

No período chuvoso, além da maior temperatura e disponibilidade de nutrientes, a condição limnológica nos bancos de ninféias caracterizou-se pela menor disponibilidade de luz devido à elevada cobertura da macrófitas no Lago das Ninféias (janeiro: 70-100\% - dados não publicados). Nestas condições ocorreu elevada participação de flagelados no início da sucessão, principalmente a r-estrategista Chromulina elegans. Esta espécie é frequentemente encontrada na comunidade perifítica dos reservatórios do PEFI, sendo associada à disponibilidade de $\mathrm{N}$ e $\mathrm{P}$ (Vercellino 2007), adição de $\mathrm{P}$ (Fermino et al. 2011) e a condição P-limitante (Ferragut \& Bicudo 2010). As crisofíceas são oportunistas e, comumente, apresentam episódios de rápido crescimento, regulados por mecanismos autogênicos, isto é, dependentes da densidade (Sandgreen 1988). Considerando o início da formação do biofilme, as algas flageladas têm a vantagem adaptativa de acessar diferentes fontes de recursos (McCormick 1996), o que pode conferir o sucesso desta forma de vida nos estádios iniciais.

Nos estádios intermediários e avançados da sucessão no período chuvoso ocorreu elevada representatividade de Zygnemaphyceae. Cosmarium regnelli e Cosmarium blyttii foram altamente correlacionadas com o período chuvoso. Considerando as condições abióticas da água e a porcentagem de cobertura de macrófitas, o sucesso das desmídias foi muito provavelmente decorrente da capacidade de adaptação do grupo à baixa disponibilidade de luz (Coesel \& Wardenaar 1990; Pillsbury \& Lowe 1999). Experimentalmente, Fermino et al. (2011) relatou no controle a alta densidade de desmídias na primavera e verão nos estádios sucessionais avançados no perifíton do Lago das Ninféias.

O processo sucessional da comunidade de algas perifíticas foi dividido em fases em função da similaridade taxonômica, substituição das descritoras da comunidade, seguida pela avaliação do crescimento algal e incremento de biomassa. Tais critérios permitiram a identificação de três fases sucessionais da comunidade de algas perifíticas no pecíolo das ninféias em cada período climático.

No período seco a fase I ( $3^{\circ}$ ao $9^{\circ}$ estádio) foi caracterizada como início da sucessão devido à pequena biomassa, baixo crescimento algal e elevada participação de r-estrategistas, tais como Synechocystis aquatilis e Chlamydomonas sordida. A fase II $\left(12^{\circ}\right.$ ao $20^{\circ}$ estádio) caracterizou-se pelo aumento exponencial de biomassa, biovolume e crescimento algal e elevada da participação de Frustulia crassinervia. A fase III ( $30^{\circ}$ estádio) caracterizou-se pela elevada perda de biomassa, redução do crescimento e dominância de Bacillariophyceae, principalmente $F$. crassinervia e N. cryptotenella.

No período chuvoso a fase I ( $3^{\circ}$ ao $6^{\circ}$ estádio) foi característica do início de colonização, ou seja, com baixa biomassa, início do crescimento algal e maior participação de r-estrategistas Chromulina elegans e Chlamydomonas sagittula. A Fase II ( $9^{\circ}$ ao $20^{\circ}$ estádio) foi caracterizada pelo aumento de biomassa e de densidade total e elevada participação de Cosmarium blyttii. A fase III ( $25^{\circ}$ estádio) caracterizou-se pela acentuada perda de biomassa e redução de crescimento algal e aumento da participação de Eunotia bilunaris.

Em suma, os resultados mostraram que as condições limnológicas mudaram sazonalmente no interior dos bancos de Nymphaea spp. e, consequentemente, influenciaram a estrutura da comunidade perifítica. A temperatura, a disponibilidade de luz e nutrientes no interior dos bancos de macrófitas foi determinante na distinção do processo de colonização e da trajetória sucessional das algas perifíticas entre os períodos climáticos. Fases sucessionais comunidade de algas perifíticas foram identificadas no período seco e chuvoso, mas a sequência de substituição de espécies foi especifica para cada período. Finalmente, concluiu-se que a estrutura da comunidade de algas perifíticas foi sensível às alterações autogênicas (sucessão), mas a escala sazonal foi a principal fonte de variabilidade da estrutura da comunidade desenvolvida em Nymphaea spp.

\section{Agradecimentos}

Os autores agradecem à Fapesp, Fundação de Amparo à Pesquisa do Estado de São Paulo, pela bolsa de iniciação científica concedida à primeira autora (Processo n ${ }^{\circ} 07 / 57729-1$ ).

\section{Referências Bibliográficas}

Ács, E. \& Kiss, K.T. 1993.Colonization process of diatoms on artificial substrates in the River Danube near Dudapest (Hungary). Hydrobiologia 269/270: 301-315.

Algarte, V.M.; Siqueira, N.S.; Murakami, E.A. \& Rodrigues, L. 2009. Effects of hydrological regime and connectivity on the interannual variation in taxonomic similarity of periphytic algae. Brazilian Journal Biology 69: 609-616.

Bicudo, C.E.M.; Carmo, C.F.; Bicudo, D.C.; Henry, R.; Pião, A.C.S.; Santos, C.M. \& Lopes, M.R.M. 2002. Morfologia e morfometria de três reservatórios do PEFI. Pp. 141-158. In: Bicudo, D.C.; Forti, M.C. \& Bicudo, C.E.M. (Orgs.). Parque Estadual das Fontes do Ipiranga: unidade de conservação ameaçada pela urbanização de São Paulo. São Paulo, Ed. Secretaria do Meio Ambiente do Estado de São Paulo. Bicudo, D.C. 1990. Considerações sobre metodologias de contagem de algas do perifíton. Acta Limnologica Brasiliensia 3: 459-475. 
Bicudo, D.C.; Necchi, O.J.R. \& Chamixaes, C.B. 1995. Periphyton studies in Brazil: present status and perspectives. In: Tundisi, J.B., Bicudo, C.E.M. \& Matsumura Tundisi, T. (Eds.). Limnology in Brazil. Rio de Janeiro, Academia Brasileira de Ciências e Sociedade Brasileira de Limnologia.

Borduqui, M.; Ferragut, C. \& Bicudo, C.E.M. 2008. Chemical composition and taxonomic structure vertical and seasonal variation of periphyton community in a shallow hypereutrophic reservoir (Garças Reservoir, São Paulo, Brazil). Acta Limnologica Brasiliensia 20: 381-392.

Borduqui, M. \& Ferragut, C. 2012. Controlling factors of successional periphytic algae in tropical hypereutrophic reservoir. Hydrobiologia 683: 109-122.

Biggs, B.J.F. 1996. Patterns in Benthic Algae in Stream. Pp. 31- 56. In: Stevenson, R.J.; Botwell, M.L. \& Lowe, R.L (Eds.). Algal ecology freshwater benthic ecosystems. San Diego, Academic Press.

Biggs, B.J.F; Stevenson, R.J. \& Lowe, R.L. 1998. A habitat matrix conceptual model for stream periphyton. Archiv für Hydrobiologie 143: 21-56.

Burkholder, J.M. 1996. Interaction of benthic algae with their substrata. Pp. 253- 298. In: Stevenson, R.J., Bothwell, M.L. \& Lowe, R.L. Algal Ecology: freshwater benthic ecosystems. San Diego, Academic Press.

Callieri, C. \& Stockner, J.G. 2002. Freshwater autotrophic picoplankton: a review. Journal of Limnology 61: 1-14.

Carpenter, S.R. \& Lodge, D.M., 1986, Effects of submersed macrophytes on ecosystem processes. Aquatic Botany 26: 341-370.

Cattaneo, A. \& Kalff, J. 1979. Primary production of algal growing on natural and artificial aquatic plants: a study of interactions between epiphytes and their substrate. Limnology and Oceanography 24: 1031- 1037.

Cattaneo, A. \& Amireault, M.C. 1992. How artificial are artificial substrate for periphyton? Journal of the North American Benthological Society 11: 44-256.

Cattaneo, A., Galanti, G., Gentinetta, S. \& Romo, S. 1998 Epiphytic algae and macroinvertebrates on submerged and floating-leaved macrophytes in an Italian lake. Freshwater Biology 39: 725-740.

Cavati, B. \& Fernandes, V.O. 2008. Algas perifíticas em dois ambientes do baixo rio Doce (lagoa Juparanã e rio Pequeno - Linhares, Estado do Espírito Santo, Brasil): variação espacial e temporal. Acta Scientiarium Biological Science Maringá 30: 439-448.

Coesel, F.P.M. \& Wardenaar, K. 1990. Growth responses of planktonic desmid species in a temperature-light gradient. Freshwater Biology 23: $551-560$

Ferragut, C. \& Bicudo, D.C. 2010. Periphytic algal community adaptive strategies in $\mathrm{N}$ and $\mathrm{P}$ enriched experiments in a tropical oligotrophic reservoir. Hydrobiologia 646: 295-309.

Fermino, F.S., Bicudo, C.E.M \& Bicudo, D.C. 2011. Seasonal influence of nitrogen and phosphorus enrichment on the floristic composition of the algal periphytic community in a shallow tropical, mesotrophic reservoir (São Paulo, Brazil). Oecologia Australis 15: 476-493.

Ferrari, F. 2010. Estrutura e dinâmica da comunidade de algas planctônicas e perifíticas (com ênfase nas diatomáceas) em reservatórios oligotrófico e hipertrófico (Parque Estadual Das Fontes do Ipiranga, São Paulo). Tese de Doutorado. UNESP, Rio Claro.

Fonseca, I.A.; Rodrigues, L. 2007. Periphytic cianobacteria in different environments from the upper Paraná river floodplain, Brazil. Acta Limnologica Brasiliensia 19(1): 53-65.

França, R.C.S., Lopes, M.R.M. \& Ferragut, C. 2011. Structural and successional variability of periphytic algal community in a Amazonian lake during the dry and rainy season (Rio Branco, Acre). Acta Amazonica 41(2): 257-266

Frost, P.C. \& Elser, J.J. 2002. Effects of light and nutrients on the net accumulation and elemental composition of epilithon in boreal lakes. Freshwater Biology 47: 173-184.

Golterman, H.L. \& Clymo, R.S. 1971. Methods for chemical analysis of freshwaters. Ed. Oxford and Edinburg, Blackwell Scientific Publications. International Biological Programmer.

Golterman, H.L., Clymo, R.S. \& Ohmstad, M.A.M. 1978. Methods for physical and chemical analysis of freshwaters. 2 ed. Blackwell Scientific Publications. Oxford, International Biological Program.

Hillebrand, H.; Durselen, C-D.; Kirschtel, D.; Pollingher, U. \& Zohary, T. 1999. Biovolume calculation for pelagic and benthic microalgae. Journal Phycology 35: 403-424.
Hoagland, K.D.; Roemer, S.C. \& Rosowski, J.R. 1982. Colonization and community structure of two periphyton assemblages, with emphasis on the diatoms (Bacillariophyceae). American Journal of Botany 69: $188-213$

Hodoki, Y. 2005 Effects of solar ultraviolet radiation on the periphyton community in lotic systems: comparison of attached algae and bacteria during their development. Hydrobiologia 534: 193-204.

Jeppesen, E.; Søndergaard, M.; Søndergaard, M \& Cristoffersen, K. 1998. The structuring role of submerged macrophytes in lakes. New York. Springer-Verlag.

Kahlert, M. 1998. C:N:P ratios of freshwater benthic algae. Archiv fur Hydrobiologie, Special Issue Advances in Limnology 51: 105-114.

Leandrini, J.A. \& Rodrigues, L. 2008. Temporal variation of periphyton biomass in semilotic environment of the upper Paraná river floodplain. Acta Limnologica Brasiliensia 20: 21-28.

Lobo, E.A. \& Leighton, G. 1986. Estructuras comunitarias de las fitocenosis planctónicas de los sistemas de desembocaduras de rios $\mathrm{Y}$ esteros de la zona central de Chile. Revista Brasileira de Botânica 22: 1-29.

Lock, M.A.; Wallace, R.R.; Costerton, J.W.; Ventullo, R.M. \& Charlton, S.E. 1984. River epilithon: toward a structural - funcional model. Oikos 42: 10-12.

Mackeret, F.J.H.; Heron, J. \& Talling, J.F. 1978. Water analysis: some revised methods for limnologists. Cumbria: Freshwater Biological. (Association Scientific Publication, 39). Kendall, Ed. Wilson, Son Ltda.

Marker, A.F.H.; Nusch, H.; Rai, H. \& Riemann, B. 1980. The measurement of photosynthetic pigments in freshwaters and standardization of methods: conclusion and recomendations. Arch. Hydrobiol. Beih. 14: 91-106.

McCormick, P.V \& Stevenson, R. J. 1991. Mechanisms of benthic algal succession in lotic environments. Ecology 72: 1835-1848.

McCormick, P.V. 1996. Resource competition and species coexistence in freshwater benthic algal assemblages. Pp.229-252. In: Stevenson, R.J., Bothwell, M.L. \& Lowe, R.L. Algal Ecology: freshwater benthic ecosystems. San Diego, Academic Press.

McCune, B. \& Mefford, M.J. 1999. PC-ORD for Windowns. Multivariate analysis of ecological data, version 4.10. Oregon, MjM Software Design.

Messyasz, B. \& Kuczynska-Kippen, N. 2006. Periphytic algal communities: A comparison of Typha angustifolla L. and Chara tomentosa L. beds in three shallow lakes (West Poland). Polish Journal of Ecology 54: 15-27.

Odum, E.G. 2001. Fundamentos de Ecologia. Lisboa, Fundação Calouste Gulbenkian.

Oliveira, D.E, Ferragut, C. \& Bicudo, D.C. 2010. Relationships between environmental factors, periphyton biomass and nutrient content in Garças Reservoir, a hypereutrophic tropical reservoir in southeastern Brazil. Lakes \& Reservoirs: Research and Management 15: 129-137.

Peterson, C.G. 1996. Responses of Benthic Algal Communities to natural physical disturbance. In: Stevenson, R.J., Bothwell, M.L. \& Lowe, R.L. Pp.375-402. Algal Ecology: freshwater benthic ecosystems. San Diego, Academic Press.

Pillsbury, R.W. \& Lowe, R.L. 1999. The response of benthic algae to manipulations of light in four acidic lakes in northern Michigan. Hydrobiologia 394: 69-81.

Rodrigues, L. \& Bicudo, D.C. 2004. Periphytic algae. Pp. 125-143. In: Thomaz, S.M.; Agostinho, A.A. \& Hahn, N.S. (Eds). The upper Paraná river and its floodplain: physical aspects, ecology and conservation. Leiden, Backhuys Publishers.

Sandgreen, C.D. 1988. The ecology of chrysophyte flagellates: their growth and perennation strategies as freshwater phytoplankton. Pp.9-104. In: Sandgreen, C.D. Growth and reproductive strategies of freshwater phytoplankton. Cambridge, Cambridge University Press.

Sartory, D.P. \& Grobbelaar, J.U. 1984. Extraction of chlorophyll a from freshwater phytoplankton for spectrophotometric analysis. Hydrobiologia 114: 177-187.

Sekar, R.; Nair, K.V.K.; Nandakumar, K.; Venugopalan, V.P. \& Rao, V.N.R 2004. Early stages of biofilm succession in a lentic freshwater environment. Hydrobiologia 512: 97-108. 
Stevenson, J.R. 1996. An in freshwater benthic habitats. Pp. 3-30. In: Stevenson, R.J. \& Bothwell, M.L. Algal Ecology:freshwater benthic ecosystems. New York, Academic Press.

Utermöhl, H. 1958. Zur Vervollkommung der quantitativen phytoplankton-methodik. Mitteilungen Internationale Vereinigung Limnologie 9: 1-38.

Vadeboncoeur, Y.; Peterson, G.; Jake, M.; Zanden, V. \& Kalff, J. 2008. Benthic algal production across lake size gradients: interactions among morphometry, nutrients, and light. Ecology 89: 2542-2552.

Valderrama, G.C. 1981. The simultaneous analysis of total nitrogen and total phosphorus in natural waters. Marine Chemical 10: 109-112.

Van Dam, H.; Mertens, A. \& Sinkeldam, J. 1994. A coded checklist and ecological indicator values of freshwater diatoms from the Netherlands. Netherlands Journal Aquatic Ecology 28: 117-133.
Vercellino, I.S. 2007. Resposta do perifíton aos pulsos de enriquecimento em níveis crescentes de fósforo e nitrogênio em represa tropical mesotrófica (Lago Ninféias, São Paulo). Tese de Doutorado, Universidade Estadual Paulista, Rio Claro.

Vercellino, I.S. \& Bicudo, D.C. 2006. Sucessão da comunidade de algas perifíticas em reservatório oligotrófico tropical (São Paulo, Brasil): Comparação entre período seco e chuvoso. Revista Brasileira de Botânica 29: 363-377.

Wehr, J.D. \& Sheath, R.G. 2003. Introduction to freshwater algae. Pp. 117-196. In: Wehr, J.D. \& Sheath, R.G. (Eds.). Freshwater algae of North America: Ecology and classification. San Diego, Academic Press.

Wetzel, R.G. 2001. Limnology: lake and river ecosystems. Third edition. San Diego, Academic Press. 